\title{
SGLT-2 inhibitors: the glucosuric antidiabetics
}

\author{
Rekha Thaddanee $^{1}$, Ajeet Kumar Khilnani ${ }^{2} *$ Gurudas Khilnani $^{3}$
}

${ }^{1}$ Department of Pediatrics, ${ }^{2}$ Department of ENT, ${ }^{3}$ Department of Pharmacology, GMERS Medical College and Hospital, Dharpur, Patan, Gujarat, India

Received: 10 May 2013

Accepted: 23 May 2013

*Correspondence to:

Dr. Ajeet Kumar Khilnani, Email: ajeetkhilnani@gmail.com

(C) 2013 Thaddanee $\mathrm{R}$ et al. This is an open-access article distributed under the terms of the Creative Commons Attribution License, which permits unrestricted use, distribution, and reproduction in any medium, provided the original work is properly cited.

\begin{abstract}
Despite availability of a number of oral antidiabetics, a sizeable population of diabetics remains uncontrolled. Thus there is growing need of new group of drugs for diabetic control. Understanding renal conservation of glucose by efficient reabsorption through sodium glucose cotransporter-2 (SGLT-2) has paved way for development of an entirely new group of drugs, the SGLT-2 inhibitors. These glucosuric antidiabetic agents have shown promise in early clinical studies. Canagliflozin is recently approved for use in diabetes alone or along with other antidiabetics. Other highly selective inhibitors undergoing various stages of clinical developments are dapagliflozin, sergliflozin, remogliflozin, ipragliflozin, empagliflozin, luseogliflozin, tofogliflozin and desoxyrhaponticin. KGA-2727 (pyrazole-O-glucoside) is the first selective SGLT-1 inhibitor undergoing intense preclinical testing. There are safety issues associated with this group like urogenital infections (fungal), weight loss, initial osmotic diuresis and increased incidence of cardiovascular events. The long term safety remains to be established. Despite these limitations, SGLT-2 inhibition offers a unique target for achieving adequate control of diabetes in adults.
\end{abstract}

Keywords: Canagliflozin, Glucosuric antidiabetics, Phlorizin, SGLT-2 inhibitors

\section{INTRODUCTION}

Diabetes mellitus is a metabolic syndrome characterized by an absolute or relative lack of insulin. Obviously, the treatment is targeted towards measures which increase insulin levels (Sulphonylureas and Meglitinides), insulin sensitivity (insulin sensitizers such as Glitazones and Biguanides) or which reduce the post prandial surge of glucose (Glycosidase inhibitors and Meglitinides). Type2 diabetes mellitus (T2DM) tends to be a progressive disease and most patients require treatment with combinations of glucose-lowering agents. The glitazones (Peroxisome Proliferator-Activated Receptor- $\alpha$ agonists, PPAR- $\alpha$ agonists) have a number of beneficial effects and are in true sense insulin sensitizers and have metabolic effects also. However, these agents have been associated with potential adverse events, such as increased cardiovascular risk, fluid retention, increased body weight, bone fractures and perhaps, bladder cancer. These agents, including metformin, are euglycemics rather than hypoglycemics (sulphonylureas).

Physiologically, oral glucose strongly stimulates release of insulin by secretion of gut hormones called incretins.
The Glucagon-Like Peptide-1 (GLP-1) is an important incretin secreted from L type of entero-endocrine cells of duodenum and jejunum. GLP-1 reduces glucagon secretion and gastric emptying and appetite. It is rapidly inactivated by dipeptidyl-peptidase-IV (DPP-IV) enzyme and thus has a very short duration of action. Analogues of GLP-1 offer attractive clinical options as antidiabetic drugs. This group of drugs is called as incretin mimetics. The incretin mimetics GLP-1 analogues (liraglutide and exenatide) are found to be useful agents in type-2 diabetes alone or along with other agents. Liraglutide has a long duration of action and is used subcutaneously as a single daily dose. Amylin analogues such as pramlintide are also euglycemic agent and in clinical trials are found to be effective in Type- 1 and Type- 2 DM but require a subcutaneous injection. Among the orally effective DPPIV inhibitors are gliptines (saxagliptin, sitagliptin and vildagliptin) which are being increasingly used alone or along with other agents for tighter control of blood glucose level. Despite availability of these agents, a large population of diabetics remains uncontrolled and thus there is a still need of new and effective agents. Understanding the physiology of glucose absorption and transport provides key steps for the identification of 
newer molecular mechanisms and enzymes which can be targeted to develop newer antidiabetic drugs. Among them are glucose transporters (GLUTs- uniporters of glucose) and a family of SLC5A1 and SLC5A2 gene products, which are sodium glucose co-transporters, SGLT-1 and SGLT-2 respectively. These proteins work by driving the uphill transport of sugar into cells down the sodium gradient across cell membranes. An SGLT-1 inhibitor would block the intestinal absorption of glucose, while an SGLT-2 inhibitor blocks reabsorption of glucose in the kidney.

This review focuses on the current role of SGLT-1 and SGLT-2 in glucose homeostasis in diabetics and impact of newly developed drugs on diabetic control.

\section{RENAL GLUCOSE HANDLING}

Glucose is the main source of energy for mammalian cells and its entry is mediated by various transporters. In August 1960, in Prague, Robert K. Crane presented for the first time his discovery of the sodium-glucose co transport as the mechanism for intestinal glucose absorption. ${ }^{1}$ Seven facilitative (GLUT 1-7) and two active glucose transporters (SGLT-1 and SGLT-2) are identified. The SGLT-1 is protein responsible for the uptake of the dietary sugars glucose and galactose from the intestinal lumen. The glycoprotein is localized in the brush border of the intestinal epithelium and contains 12 membrane spans. SGLT-1 uses the electrochemical gradient of two sodium ions to transport one glucose molecule. Both the sodium glucose co-transporters SGLT-1 and SGLT-2 are also expressed in kidneys. ${ }^{2}$

Glucose is easily filtered and reabsorbed but is not secreted from tubules. The reabsorption of filtered load of glucose occurs mostly in proximal tubules (S1 and S2 segments) and is very efficient normally (98\%) to conserve glucose in body. Almost the entire filtered load (140 grams) undergoes complete tubular reabsorption. The S1 and S2 segments of proximal convoluted tubules (PCT) show low affinity but high capacity for glucose reabsorption. SGLT-2 is mainly expressed on the brush borders of cells of these segments of PCT. SGTL-2 cotransports glucose and sodium using sodium gradient created by $\mathrm{Na}^{+} / \mathrm{K}^{+}$ATPase pump. The $\mathrm{Na}+\mathrm{K}+$ ATPase pump on the basolateral membrane of the proximal tubule cell uses ATP to move three sodium outward into the blood, while bringing in two potassium. This creates a downhill sodium gradient inside the proximal tubule cell in comparison to both the blood and the tubule. The SGLT proteins use the energy from this downhill sodium gradient created by the ATPase pump to transport glucose across the apical membrane against an uphill glucose gradient. Once inside cells, the GLUT uniporters further transport glucose from basolateral membrane to peritubular capillaries. The SGLT-1 is also expressed in cells of S3 segment of PCT and reabsorbs remaining glucose not reabsorbed in $\mathrm{S} 1$ and $\mathrm{S} 2$ segments. In diabetics the SGLT-2 is upregulated because of persistent hyperglycemia. A similar upregulation of GLUT-2 is also reported in diabetics. ${ }^{3}$

\section{PHLORIZIN}

A glucoside obtained from apple bark in which glucose is joined with two aromatic rings with the help of an alkyl group. It is an experimental agent used to study glucose homeostasis. Von Mering, as early as 1886, observed that phlorizin produced glucosuria. Later, it was shown to be high-affinity competitive inhibitor of sodium-glucose transport in renal and intestinal epithelial cells. ${ }^{4}$ Administration of phlorizin in rats causes heavy glucosuria and glucose (and galactose) malabsorption. It was shown to reduce elevated blood glucose in streptozotocin induced diabetes in rats. A metabolite of phlorizin is T-1095 and it was shown to improve hyperglycemia and insulin resistance in diabetes. Phlorizin is not suitable clinically because of nonselective inhibition of SGLT-1 and SGLT-2, and low oral bioavailability. However, it has paved way for development of an entirely new group of drugs called as SGLT-2 inhibitors.

\section{SGLT-2: A NEW TARGET FOR DEVELOPMENT OF DIABETIC AGENTS}

From the above observations it can be hypothesized that inhibition of SGLT-2 may provide an attractive, insulinindependent target for increasing glucose excretion. If renal handling of glucose (mediated by SGLT-2) is manipulated by such agent, then it could be possible to use such an agent for control of diabetes. Several structural analogues of phlorizin have been synthesized and screened for evaluating SGLT-2 inhibiting activity using in vitro and renal brush border membrane assays. Results from preclinical studies have shown that they increase glucose excretion and normalize plasma glucose in experimental diabetic models. Initial clinical data are promising and suggest that SGLT-2 inhibitors may be a new therapeutic option for treating type-2 diabetes mellitus. Truly they are glucosuric and antihyperglycemic agents. Highly selective inhibitors undergoing various stages of clinical developments are dapagliflozin- a Caryl glucoside, sergliflozin- a benzylphenol glucoside, remogliflozin- a benzylpyrazole glucoside, ipragliflozin, empagliflozin, luseogliflozin, canaglifozin- a C-glucoside with a thiophene ring recently approved by FDA, tofogliflozin and desoxyrhaponticin. KGA-2727 (pyrazole-O-glucoside) is the first selective SGLT-1 inhibitor undergoing intense preclinical testing.

\section{GENERAL CHARACTERISTICS OF GLUCOSIDE SGLT-2 INHIBITORS}

In general all these compounds cause renal glucosuria and reduce elevated blood glucose in fasting and post prandial states in a dose dependent manner in rodents, mice and humans. ${ }^{6}$ In early clinical trials a reduction in $\mathrm{HbA1c}$ is also reported. The antihyperglycemic effect is 
insulin independent. These agents may also reverse $\beta$-cell dysfunction and insulin resistance in T2DM. An expected long-term benefit is improved glucose intolerance and reduced insulin resistance thus prevention of diabetic complications such as neuropathy. ${ }^{7}$ Other favorable effects of SGLT-2 inhibitors include a reduction in both, body weight and blood pressure. Therefore, these agents can be used alone or along with other antidiabetic agents with different mechanisms of action. These agents usually do not cause hypoglycemia because of two reasons. One, activity of SGLT-1 in distal part of PCT (Segment-3) allows reabsorption of some glucose and two, intestinal SGLT-1 is not inhibited thus absorption of glucose is continued. Overall, because of glucosuria, a negative energy balance leads to weight loss and improved insulin sensitivity. The latter effect is due to removal of gluco-toxic effect on the $\beta$-cells. These agents do not inhibit GLUT induced glucose absorption across biomembranes. Therefore, glucose supply to the vital tissues such as brain, liver, and muscle remains intact. Glucosuria initially may cause osmotic diuresis, which may be beneficial in some cases but may evoke secretion of diabetogenic counter regulatory hormones (cortisol, glucagon and catecholamines) due to volume contraction. The continued presence of glucose in urine may predispose to urinary tract infection. This, along with bladder dysfunction in diabetics, acts as a risk factor for UTI, especially fungal infections. These safety issues need to be further evaluated.

\section{Sergliflozin (KTG-1251)}

It is inactive by itself and is converted to active compound which inhibits SGLT-2 more selectively (7 times) than SGLT-1. In early clinical studies, it lowers postprandial blood glucose in rodent models by inducing glucosuria. The effect is independant of insulin secretion. In healthy obese volunteers, 500-1000 mg daily dose of sergliflozin reducued weight compared to placebo treated cohort. In diabetics sergliflozin reduced mean plasma glucose and caused dose dependent glucosuria. Adverse effects noted were headache, dyspepsia and sore throat. ${ }^{8}$

\section{Dapaglifozin}

This agent has favorable pharmacokinetic profile in humans. It can be given as a single daily dose. Like sergliflozin, it also produces dose dependent glucosuria. No effect on urine and serum electrolytes has been noted in a study. ${ }^{9}$ Two episodes of hypoglycemia were reported. In a phase-IIb study on 47 diabetics, a dose of 25-100 mg dapagliflozin, given as a single daily dose inhibited renal reabsorption of glucose by $40 \%$. Adverse effects reported were nausea, constipation, vulvovaginal mycotic infection. In a multiple dose comparative study with metformin, dapagliflozin reduced hyperglycemia and caused weight loss comparable to metformin. Genital infections were common in dapagliflozin group. ${ }^{10} \mathrm{~A}$ comparative study versus glipizide showed similar 52week glycemic efficacy, but reduced weight and produced less hypoglycemia than glipizide in type-2 diabetes inadequately controlled with metformin. ${ }^{11}$ Longterm studies are required to further evaluate genital and urinary tract infections with SGLT-2 inhibitors. In another study, dapagliflozin was given for 48 weeks in patients on high dose of insulin. It improved glycemic control, stabilized insulin dosing, and reduced weight without increasing major hypoglycemic episodes in patients with inadequately controlled type- 2 diabetes mellitus. Compared with the placebo group, patients in the pooled dapagliflozin groups had a higher rate of hypoglycemic episodes (56.6\% vs. $51.8 \%$ ), events suggesting genital infection (9.0\% vs. $2.5 \%)$, and events suggesting urinary tract infection $(9.7 \%$ vs. $5.1 \%) .{ }^{12}$ In another study, dapagliflozin added to glimepiride in patients with uncontrolled T2DM significantly improved $\mathrm{HbA} 1 \mathrm{c}$, reduced weight and was generally well tolerated, although events suggestive of genital infections were reported more often in patients receiving dapagliflozin. ${ }^{13}$ In a long-term trial, dapagliflozin (2.5-5.0 mg/day) added to metformin for 102 weeks enabled sustained reductions in HbA1c, fasting plasma glucose (FPG), and weight without increased risk of hypoglycemia in patients with type-2 diabetes who were inadequately controlled on metformin alone. Evidence suggestive of urinary tract infection was reported in $8.0 \%$ to $13.3 \%$ of dapagliflozin patients and $8.0 \%$ of placebo patients, with one related discontinuation (dapagliflozin $2.5 \mathrm{mg}$ ).$^{14}$ Incidence of hypoglycemia is lower than sulphonylurea and occurs particularly when given along with insulin.

\section{Remogliflozin}

Remogliflozin ebonite salt is metabolized to remogliflozin in body. Structurally it is dissimilar to other SGLT-2 inhibtors, being a benzylpyrazole glucoside. It has shown antihyperglycemic and glucosuria effects in rodents, mice and humans. In T2DM, reduction in hyperglycemia and HbA1c levels is reported. ${ }^{15}$

\section{Ipragliflozin}

It is a SGLT-2 inhibitor in Phase 3 clinical development for the treatment of type-2 diabetes mellitus (T2DM). ${ }^{16}$

\section{Empagliflozin}

It is another highly selective SGLT-2 inhibitor being intensely evaluated in diabetes. Oral administration of empagliflozin at doses of 10,25 or 100 mg once daily over 28 days resulted in significant increases in urinary glucose excretion (UGE) and reductions in blood glucose compared with placebo, and were well tolerated in patients with type 2-diabetes. The most frequently reported adverse effects were pollakiuria $(10.3 \%)$, nasopharyngitis $(9.0 \%)$, constipation $(9.0 \%)$ and headache $(7.7 \%) .{ }^{17}$ No adverse drug interactions were found with oral contraceptive pills containing ethinyl estradiol and levonorgestrel. ${ }^{18}$ No dose adjustment 
of empagliflozin is required when coadministered with ramipril, digoxin or verapamil. ${ }^{19}$ Empagliflozin reduces high glucose induced inflammatory and fibrotic markers by blocking glucose transport and did not induce a compensatory increase in SGLT-1/GLUT-2 expression. It attenuates Toll-like receptor-4 expression, nuclear deoxyribonucleic acid binding for nuclear factor kappa $\mathrm{B}$ $(\mathrm{NF}-\mathrm{\kappa B})$ and activator protein 1 induced collagen IV expression as well as interleukin- 6 secretion suggesting a renoprotective effect related to a reduction of glucotoxicity. ${ }^{20}$

\section{Canagliflozin}

Nomura et al discovered that C-glucosides bearing a heteroaromatic ring formed metabolically more stable inhibitors for sodium-dependent glucose cotransporter-2 (SGLT-2) than the O-glucosides. A novel thiophene derivative 4b-3 (canagliflozin) was a highly potent and selective SGLT-2 inhibitor and showed pronounced antihyperglycemic effects in high-fat diet fed KK (HF-KK) mice. ${ }^{21}$

Canagliflozin improves glycemic control in an insulinindependent fashion through inhibition of glucose reuptake in the kidney. It offers a relatively modest reduction in $\mathrm{HbA} 1 \mathrm{c}$, FPG, and postprandial plasma glucose (PPG) (glycemic control). It has a low incidence of hypoglycemia and a reduction in body weight. Dose adjustment may be recommended in the elderly, those on loop diuretics, and those with an estimated glomerular filtration rate $(e G F R)<60 \mathrm{ml} / \mathrm{min} / 1.73 \mathrm{~m}^{2}$. There is a rise in low-density lipoprotein cholesterol (LDL-C) and the odds of heart attack and stroke. It is recently approved by the US Food and Drug Administration in April 2013 and is undergoing evaluation by the European Medicines Agency. ${ }^{22}$

Schernthaner et al (2013) evaluated the efficacy and safety of canagliflozin compared with sitagliptin in subjects with T2DM inadequately controlled with metformin plus sulfonylurea in a 52-week, randomized, double-blind, active-controlled, phase 3 study. Subjects using metformin plus sulfonylurea $(\mathrm{N}=755)$ received canagliflozin $300 \mathrm{mg}$ or sitagliptin $100 \mathrm{mg}$ daily. Primary end point was change from baseline in $\mathrm{HbA} 1 \mathrm{C}$ at 52 weeks. Secondary end points included change in fasting plasma glucose (FPG) and systolic blood pressure (BP), and percent change in body weight, triglycerides, and HDL cholesterol. Safety was assessed based on adverse event reports. At 52 weeks, canagliflozin $300 \mathrm{mg}$ demonstrated noninferiority and, in a subsequent assessment, showed superiority to sitagliptin $100 \mathrm{mg}$ in reducing $\mathrm{HbA1C}(-1.03 \%$ [-11.3 $\mathrm{mmol} / \mathrm{mol}]$ and $-0.66 \%$ [-7.2 $\mathrm{mmol} / \mathrm{mol}]$, respectively. Greater reductions in FPG, body weight, and systolic BP were observed with canagliflozin versus sitagliptin $(\mathrm{P}<$ 0.001). Overall adverse event rates were similar with canagliflozin $(76.7 \%)$ and sitagliptin (77.5\%); incidence of serious adverse events and adverse event related discontinuations was low for both groups. Higher incidences of genital mycotic infections and osmotic diuresis-related adverse events were observed with canagliflozin, which led to one discontinuation. Hypoglycemia rates were similar in both groups. Authors concluded that canagliflozin may be a new therapeutic tool providing better improvement in glycemic control and body weight reduction than sitagliptin, but with increased genital infections in subjects with type-2 diabetes using metformin plus sulfonylurea. ${ }^{23}$ It appears that canagliflozin also inhibits intestinal SGLT-1 protein. Canagliflozin reduces postprandial plasma glucose and insulin by increasing urinary glucose excretion (via renal SGLT-2 inhibition) and also delaying rate of appearance of glucose in blood after an oral load, likely due to reduced absorption by intestinal SGLT-1 inhibition. ${ }^{24}$

In another randomized, double-blind, placebo-controlled, phase 3 efficacy and safety trial, subjects $(\mathrm{N}=269)$ received canagliflozin $100 \mathrm{mg}$ or $300 \mathrm{mg}$ or placebo daily. The primary efficacy endpoint was change from baseline in HbA1c at 26 weeks. Secondary endpoints were change in fasting plasma glucose (FPG) and proportion of subjects reaching $\mathrm{HbA} 1 \mathrm{c}<7.0 \%$. Safety was assessed based on adverse event reports; renal safety parameters (e.g. eGFR, blood urea nitrogen and albumin/creatinine ratio) were also evaluated. Canagliflozin 100 and $300 \mathrm{mg}$ doses significantly reduced blood glucose and $\mathrm{HbA1c}$. Overall adverse event rates were similar for canagliflozin 100 and $300 \mathrm{mg}$ and placebo $(78.9,74.2$ and $74.4 \%)$. Slightly higher rates of urinary tract infections and adverse events related to osmotic diuresis and reduced intravascular volume were observed with canagliflozin $300 \mathrm{mg}$ compared with other groups. Transient changes in renal function parameters that reverted towards baseline over 26 weeks were observed with canagliflozin. Authors concluded that canagliflozin improved glycaemic control and was generally well tolerated in subjects with T2DM and Stage 3 chronic kidney disease (CKD). ${ }^{25}$

Canagliflozin (Invokana) can be used as monotherapy or along with other oral antidiabetic agents. It should be taken early in the morning before breakfast. The dose needs to be regulated in kidney disease and is avoided if eGFR is less than $40 \mathrm{ml} / \mathrm{min} / 1.73 \mathrm{~m}^{2}$. It can cause hyperkalemia, mycotic vaginitis and balanitis (10\%), urinary tract infection (5\%). There is some evidence that it may produce more cardiovascular events. The results of five ongoing post approval trials will clarify these safety issues.

\section{SOME MORE CONCERNS}

There is a theoretical possibility of loss of other molecules whose molecular weight equals glucose. In addition, persistent glucosuria could be detrimental to health because of loss of energy. There are two groups of maturity onset diabetes; obese and lean diabetics. The SGLT-2 inhibitors are beneficial in obese diabetics, but 
what place these agents have for the control of diabetes in lean adults remains to be known. The incidence of urogenital infections on long term use remains to be evaluated.

\section{CONCLUSION}

Renal SGLT-2 inhibition is a clinically useful strategy for control of diabetes. A number of agents having glucoside moiety are being developed and are in various stages of clinical testing. Canagliflozin is approved for use currently and others are likely to be approved soon. While these agents have shown promising results in short term trials, long term effect of this new group of drugs remains to be known.

\section{REFERENCES}

1. Wright EM, Turk E. The sodium/glucose cotransport family SLC5. Pflugers Arch 2004;447 (5):510-8.

2. Wood IS, Trayhum P. Glucose transporters (GLUT and SGLT): Expanded families of sugar transport proteins. Br J Nutr 2003;89:3-9.

3. Freitas HS, Ahne GF, Melo MM, Okamoto M, oliviera-Souza M, Bordin S. Na+-Glucose transporter-2 messanger ribonucleic acid expression in kidney of diabetic rats correlates with glycemic levels. Involvement of hepatocute nuclear factor-1a expression and activity. Endocrinol 2008;149: 717-24.

4. Ehrenkranz JR, Lewis NG, Kahn CR, Roth J. Phlorizin: A review. Diabetes Metab Res Rev. 2005;21:31-8.

5. Basile J. New approach to glucose control in type 2 diabetes: the role of kidney sodium-glucose cotransporter 2 inhibition. Postgrad Med. 2011; 123:38-45.

6. Raskin P. Sodium-glucose cotransporter inhibition: Therapeutic potential for the treatment of type 2 diabetes mellitus. Diabetes Metab Res Rev. 2013 Mar 5. doi: 10.1002/dmrr.2403. [Epub ahead of print].

7. Chao EC, Henry RR. SGLT2 inhibition--a novel strategy for diabetes treatment Nat Rev Drug Discov. 2010 Jul;9(7):551-9.

8. Katsuno K, Fujimori Y, Takemura Y, Hiratochi M, Itoh F, Komatsu Y, Fujikura H, Isaji M. Sergliflozin, a novel selective inhibitor of lowaffinity sodium glucose cotransporter (SGLT2), validates the critical role of SGLT2 in renal glucose reabsorption and modulates plasma glucose level. J Pharmacol Exp Ther. 2007;320:323-30.

9. Clar C, Gill JA, Court R, Waugh N. Systematic review of SGLT2 receptor inhibitors in dual or triple therapy in type 2 diabetes. BMJ Open. 2012 Oct 18;2(5). pii: e001007. doi: 10.1136/bmjopen-2012-001007. Print 2012.

10. Nauck MA, Del Prato S, Meier JJ, Durán-García S, Rohwedder K, Elze M, Parikh SJ. Dapagliflozin versus glipizide as add-on therapy in patients with type 2 diabetes who have inadequate glycemic control with metformin: a randomized, 52-week, double-blind, active-controlled noninferiority trial. Diabetes Care. 2011 Sep;34:2015-22.

11. Nauck M, Del Prato S. SGLT-2inhibitor dapagliflozin: new treatment approach for diabetes type 2 - new achievements, but also new questions!. Dtsch Med Wochenschr. 2013;138 Suppl 1:S4-6.

12. Wilding JP, Woo V, Soler NG, Pahor A, Sugg J, Rohwedder K, Parikh S; Long-term efficacy of dapagliflozin in patients with type 2 diabetes mellitus receiving high doses of insulin. Dtsch Med Wochenschr. 2013;138 Suppl 1:S27-39.

13. Strojek K, Yoon KH, Hruba V, Elze M, Langkilde AM, Parikh S. Effect of dapagliflozin in patients with type 2 diabetes who have inadequate glycaemic control with glimepiride. Dtsch Med Wochenschr. 2013;138 Suppl 1:S16-27.

14. Bailey CJ, Gross JL, Hennicken D, Iqbal N, Mansfield TA, List JF. Dapagliflozin add-on to metformin in type 2 diabetes inadequately controlled with metformin: a randomized, doubleblind, placebo-controlled 102-week trial. BMC Med. 2013 Feb 20;11:43.

15. Fujimori Y, Katsuno K, Nakashima I, IshikawaTakemura Y, Fujikura H, Isaji M. Remogliflozin etabonate, in a novel category of selective lowaffinity sodium glucose cotransporter (SGLT2) inhibitors, exhibit antidiabetic efficacy in rodent models. J Pharmacol Exp Ther. 2008 ;327:268-76.

16. Kurosaki E, Ogasawara H. Ipragliflozin and other sodium-glucose cotransporter-2 (SGLT2)

inhibitors in the treatment of type 2 diabetes: Preclinical and clinical data. Pharmacol Ther. 2013 Apr 4. pii: S0163-7258(13)00082-X.

17. Heise T, Seewaldt-Becker E, Macha S, Hantel S, Pinnetti S, Seman L, Woerle HJ. Safety, tolerability, pharmacokinetics and pharmaco dynamics following 4 weeks' treatment with empagl iflozinonce daily in patients with type 2 diabetes. Diabetes Obes Metab. 2013 Jan 28. doi: 10.1111/dom.12073. [Epub ahead of print].

18. Macha S, Mattheus M, Pinnetti S, Woerle HJ, Broedl UC. Effect of Empagliflozin on the Steady-State Pharmacokinetics of Ethinylestradiol and Levonorgestrel in Healthy Female Volunteers. Clin Drug Investig. 2013 Mar 20. [Epub ahead of print].

19. Macha S, Sennewald R, Rose P, Schoene K, Pinnetti S, Woerle HJ, Broedl UC. Lack of clinically relevant drug-drug interaction between empagliflozin, a sodium glucose cotransporter 2 inhibitor, and verapamil, ramipril, or digoxin in healthy volunteers. Clin Ther. 2013 Mar;35:22635.

20. Panchapakesan U, Pegg K, Gross S, Komala MG, Mudaliar H, et al. Effects of SGLT2 Inhibition in Human Kidney Proximal Tubular Cells- 
Renoprotection in Diabetic Nephropathy? PLoS ONE 8(2): e54442.

21. Nomura S, Sakamaki S, Hongu M, Kawanishi E, Koga Y, Sakamoto T, Yamamoto Y, Ueta K, Kimata H, Nakayama K, Tsuda-Tsukimoto $\mathrm{M}$. Discovery of canagliflozin, a novel C-glucoside with thiophene ring, as sodium-dependent glucose cotransporter 2 inhibitor for the treatment of type 2 diabetes mellitus. J Med Chem. 2010 Sep 9;53:6355-60.

22. Lamos EM, Younk LM, Davis SN. Canagliflozin , an inhibitor of sodium-glucose cotransporter 2, for the treatment of type 2 diabetes mellitus. Expert Opin Drug Metab Toxicol. 2013 Apr 17. [Epub ahead of print].

23. Schernthaner G, Gross JL, Rosenstock J, Guarisco $\mathrm{M}, \mathrm{Fu} \quad \mathrm{M}$, Yee J, Kawaguchi M, Canovatchel W, Meininger G. Canagliflozin Compared With
Sitagliptin for Patients With Type 2 Diabetes Who Do Not Have Adequate Glycemic Control With Metformin Plus Sulfonylurea: A 52-week randomized trial. Diabetes Care. 2013 Apr 5. [Epub ahead of print] PMID:23564919).

24. Polidori D, Sha S, Mudaliar S, Ciaraldi TP, Ghosh A, Vaccaro N, Farrell K, Rothenberg P, Henry RR.C anagliflozin Lowers Postprandial Glucose and Insuli $\mathrm{n}$ by Delaying Intestinal Glucose Absorption in Add ition toIncreasing Urinary Glucose Excretion:

Results of a randomized, placebo-controlled study. Diabetes Care. 2013 Feb 14. [Epub ahead of print].

25. Yale JF, Bakris G, Cariou B, Yue D, David-Neto E, Xi L, Figueroa K, Wajs E, Usiskin K, Meininger G. Efficacy and safety of canagliflozin in subjects with type 2 diabetes and chronic kidney disease. Diabetes Obes Metab. 2013;15:463-73.

doi:10.5455/2319-2003.ijbcp20130801

Cite this article as: Thaddanee R, Khilnani AK, Khilnani G. SGLT-2 inhibitors: the glucosuric antidiabetics. Int J Basic Clin Pharmacol 2013;2:34752. 